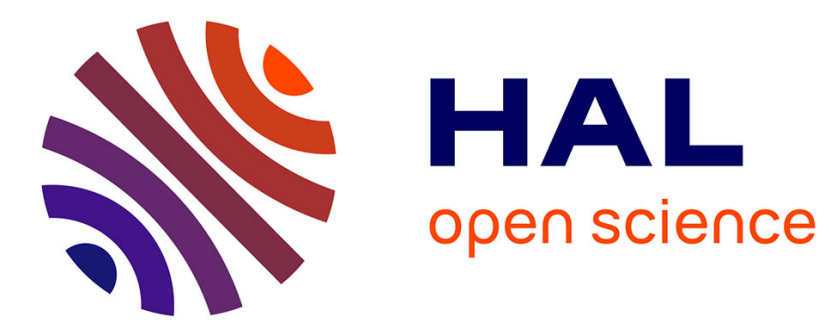

\title{
Optimisation de la quantification des flux de matière en suspension d'un cours d'eau alpin: l'Isère à Grenoble (France).
}

\author{
Dominique Dumas
}

\section{- To cite this version:}

Dominique Dumas. Optimisation de la quantification des flux de matière en suspension d'un cours d'eau alpin : l'Isère à Grenoble (France).. Comptes Rendus Géoscience, 2004, 336 (13), pp.1149-1159. halshs-00324616

\author{
HAL Id: halshs-00324616 \\ https://shs.hal.science/halshs-00324616
}

Submitted on 7 Apr 2009

HAL is a multi-disciplinary open access archive for the deposit and dissemination of scientific research documents, whether they are published or not. The documents may come from teaching and research institutions in France or abroad, or from public or private research centers.
L'archive ouverte pluridisciplinaire HAL, est destinée au dépôt et à la diffusion de documents scientifiques de niveau recherche, publiés ou non, émanant des établissements d'enseignement et de recherche français ou étrangers, des laboratoires publics ou privés. 


\title{
Optimisation de la quantification des flux de matière en suspension d'un cours d'eau alpin : l'Isère à Grenoble (France)
}

\author{
Dominique Dumas \\ Institut de Géographie Alpine, 14 bis, Avenue Marie Reynoard, 38100 Grenoble, France
}

\begin{abstract}
Résumé
Cette étude cherche à cerner les flux de matière en suspension de l'Isère, l'un des plus important cours d'eau des Alpes du Nord, à Grenoble. Depuis 1994, et surtout 1996, des prélèvements systématiques en rive gauche permettent de suivre en un point les variations de la matière en suspension de ce cours d'eau. Mais à partir d'une seule mesure, parfois peu représentative de l'ensemble des concentrations observables au sein de la section, il reste encore difficile d'estimer précisément les flux de MES. Les résultats de cette étude montrent notamment l'apparition successive de plusieurs gradients de turbidité, dont l'occurrence s'explique en grande partie par la dynamique hydrologique et hydrosédimentaire de ce cours d'eau, L'estimation de ces gradients permet ainsi d'extrapoler plus aisément les mesures ponctuelles et, dès lors, de définir quotidiennement les quantités de matières en suspension exportées par l'Isère. Depuis 1996, les résultats montrent un flux spécifique de l'Isère à Grenoble très élevé, variant entre 124 et plus de $460 \mathrm{t} \mathrm{km}^{-2}$ $\mathrm{an}^{-1}$.
\end{abstract}

\begin{abstract}
Optimization of suspended matter-fluxes quantification of an Alpine river: the Isère at Grenoble (France). This study seeks to determine suspended-matter flux of the Isère at Grenoble, one of the most important rivers of the northern French Alps. Since 1994, and especially 1996, systematic samplings of left bank make it possible to follow in one point the variations of the suspended particles and matter (SPM) of this river. But from a single measurement, sometimes not very representative of the whole observable concentrations within the section, it remains still difficult to estimate precisely the SPM flux. The results of this study show in particular the successive appearance of several turbidity gradients, whose occurrence is explained mainly by the hydrological and hydro-sedimentary dynamics of this river. The estimate of these gradients thus makes it possible to extrapolate specific measurements more easily and consequently to daily define the quantities of suspended matter exported by the Isère. Since 1996, the results show a very high flow specific of Isère, at Grenoble, variable between 124 and more than $460 \mathrm{t} \mathrm{km}^{-2} \mathrm{yr}^{-1}$.
\end{abstract}

Mots-clés : Alpes ; bassin versant de l'Isère moyenne ; gradients de turbidité ; flux sédimentaire ; France

Keyword : French Alps ; medium Isère watershed basin ; turbidity gradients ; sedimentary flow ; France

\section{Abridged English version}

\section{Introduction}

The Isère remains a particularly revealing witness of hydro-sedimentary operation of the great alpine rivers, with very high specific rates of erosion and strong temporal variability of the sediment concentrations. Since 1994, regular sampling carried out on Isère in Grenoble makes it possible to follow the variations of the suspended particles and matter (SPM) in one point of the section. Is it possible for all that to extrapolate these specific values to the whole section?

More than ten complete gauges were carried out under varied hydrological conditions within the framework of this study, The obtained SPM profiles from complete gauging show a great variability of the turbidity gradients within the section. Different multivariate regressive models were tested in order to determine the variables likely to better explain the distribution of the concentrations within the section of Isère. We thus propose a SPM fluxes estimation model considering the measurements in one point but also the hydrological and hydro-sedimentary dynamics of Isère. 


\section{The watershed basin of Isère}

The medium and higher Isère basin $\left(5570 \mathrm{~km}^{2}\right)$ is located in France, in the northern Alps (Fig. 1), In Grenoble, with a mean annual discharge of approximately $200 \mathrm{~m}^{3} \mathrm{~s}^{-1}$, the Isère is the one of the most important Alpine and tributary rivers of the Rhone. We evaluate the average low water level at $70 \mathrm{~m}^{3} \mathrm{~s}^{-1}$, the decennial water rise at approximately $950 \mathrm{~m}^{3} \mathrm{~s}^{-1}$ and the centennial flood at more than $1600 \mathrm{~m}^{3} \mathrm{~s}^{-1}$.

\section{Methodology}

On the Grenoble university campus, 17 complete gauges of the solid discharge were carried out. All these gauges were carried out simultaneously with a gauging of the liquid flow by velocity measurement in different points of the section $[5,7,11,15,18,28]$. Each gauging of the solid discharge takes place over $2 \mathrm{~h}$ during which the level of Isère evolves, sometimes in really important proportions (Table 1).

\section{Results presentation}

The obtained SPM profiles are extremely variable and not always show more important suspended matter concentrations in the vicinity of the bottom. We find, in a more or less clear way, four different distribution families of the concentrations (Fig. 2). The most precisely possible quantification of these sediments flows requires seeking parameters being able to intervene in the passage of one concentrations distribution mode to another. When the solid discharge is approximated from only one single sample (SPMfixed), the recorded value can sometimes be quite close to the mean concentration $(\mathrm{Cm})$ but sometimes definitely underestimated or, on the contrary, over-estimated (Fig. 2). Correction of the values measured in one point directly using a single regressive model is not therefore very certain, even if this presents a good correlation coefficient for the Isère (Eq. (1)) :

$\mathrm{C}_{\mathrm{m}}=0.832 \mathrm{SPM}_{\mathrm{fixed}}+18.46$

$\mathrm{R}^{2}=0.94(1)$

For each complete gauging, we described the concentration distribution from different statistical parameters resulting from the hydrodynamic and hydro-sedimentary characteristics of Isère (Table 2). Different multivariate regressive models were tested in order to determine the variables likely to better explain this parameter. Three variables play, in a highly significant way, a role in the nature of the concentrations distribution within the section (Eq. (3)) : flow-time variation $\left(T_{Q}\right)$, concentration average time variation ( $T_{\text {SPM }}$ ) and water instantaneous mean velocity $\left(\mathrm{V}_{\mathrm{M}}\right)$. The real average concentration within the section is then approximated from Eq. (4) :

$\mathrm{C}_{\mathrm{m}}=0.926 \mathrm{SPM}_{\mathrm{fixed}}-0.445 \mathrm{~T}_{\mathrm{Q}}+10.539 \mathrm{~T}_{\mathrm{SPM}}-446.41 \mathrm{~V}_{\mathrm{M}}+502.2$

Beyond a high coefficient of correlation $\left(R^{2}=0.987\right)$, the consideration of hydrological dynamics and concentrations, easily known with uninterrupted measurements, makes it possible to suppose a more robust SPM flows quantification from a unique sample.

\section{Conclusion}

The Isère Basin's mountainous context and its regime, disturbed by many hydroelectric installations, generate, during one year, SPM flows marked by a succession of peaks. These strong temporal fluctuations are accompanied by a great variability of the turbidity gradients within this river section. Also flow quantification on this type of river is extremely delicate. The consideration of hydrological parameters and the SPM flows dynamics appreciably improves distributions knowledge and thus offers a more robust extrapolation of a single-point measurement. For the case of the Isère, calculated SPM flows show a great annual variability according to the hydraulicity of the river. The values spread out from 750000 tons in 1997, to nearly 2.6 million tons in 1999, which then represents half of the average annual solid discharge of the Rhone at its mouth ( 5 to $6 \mathrm{Mt} \mathrm{yr}^{-1}$ ) [25]. The specific flux of Isère at Grenoble varies, according to years, between 124 and more than $460 \mathrm{t} \mathrm{km}^{-2} \mathrm{yr}^{-1}$, which is considerable, but in part join the East-Pyrenean edge zonation, with the proposed specific erosion rate of 250 to $350 \mathrm{t} \mathrm{km}^{-2} \mathrm{yr}^{-1}[26,27]$. 


\section{Introduction}

Depuis 1994, des prélèvements réguliers effectués sur l'Isère à Grenoble permettent de suivre les variations de la matière en suspension (MES) et de la matière dissoute (MD) en un point de la section. Ces mesures systématiques sont effectuées par l'intermédiaire de deux capteurs infrarouges (turbidimètres) et d'un préleveur automatique fixé sur la berge. Est-il possible pour autant d'extrapoler ces valeurs ponctuelles à l'ensemble de la section ? La détermination de la concentration moyenne de matière en suspension d'un cours d'eau, à partir d'un seul point de mesure, demeure toujours délicate, car les concentrations présentent sur une section transversale une variabilité spatiale parfois non négligeable. Pour cette raison notamment, l'estimation des flux de MES à partir d'un nombre réduit d'échantillons, ou d'échantillons de surface, intègre toujours une grande incertitude $[6,8,22]$. La connaissance précise des flux de MES nécessite, en toute rigueur, un jaugeage complet toujours difficile à mettre en place sur des cours d'eau importants. À cette opération délicate est substitué le plus souvent un prélèvement unique, jugé - à tort ou à raison - comme représentatif des concentrations dans la section. L'extrapolation d'un point de mesure peut être améliorée avec la détermination des gradients de turbidité [31]. Mais quelle est la variabilité temporelle de ces gradients?

En 1998, six jaugeages complets ont été réalisés à l'initiative de J.-L. Peiry [30]. Afin de compléter ces observations, onze jaugeages complets ont été de nouveau effectués [10]. Les données acquises en 2001 et 2002 permettent, d'une part, de confronter les mesures ponctuelles aux concentrations moyennes réelles et, d'autre part, de proposer un modèle d'estimation des flux de MES. Ce dernier intègre les mesures effectuées en un point mais aussi les gradients de turbidité estimés à partir de la dynamique hydrologique et hydrosédimentaire de l'Isère. La quantification des flux annuels est ainsi évaluée pour les années 1996 à 2002, années pour lesquelles des mesures en continu des concentrations ont été effectuées. Les mesures antérieures au $1^{\text {er }}$ septembre 1999 ont été fournies par J.-L. Peiry.

\section{Le bassin de l'Isère}

Le bassin de l'Isère moyenne et supérieure $\left(5570 \mathrm{~km}^{2}\right)$ est situé dans les Alpes du Nord (Fig. 1). II s'inscrit dans les chaînes intra-alpines, en intégrant les massifs de la Vanoise, du Beaufortin, de Belledonne et reçoit des affluents torrentiels qui descendent du rebord oriental des Préalpes du Nord (massifs des Aravis, des Bauges et de la Chartreuse). Le régime de l'Isère à Grenoble, à l'extrémité méridionale du sillon alpin, est connu depuis plus de 150 ans. Avec un module annuel d'environ $200 \mathrm{~m}^{3} \mathrm{~s}^{-1}$, I'Isère est l'un des plus importants cours d'eau alpins et affluents du Rhône. On évalue l'étiage moyen à $70 \mathrm{~m}^{3} \mathrm{~s}^{-1}$, la crue décennale à environ $950 \mathrm{~m}^{3} \mathrm{~s}^{-1}$ et à plus de $1600 \mathrm{~m}^{3} \mathrm{~s}^{-1}$ la crue centennale. Son régime hydrologique, marqué par la composante nivo-glaciaire, s'explique pour l'essentiel par le caractère montagneux de son bassin, dont plus de $70 \%$ de la superficie sont situés au-dessus de $1000 \mathrm{~m}$ d'altitude.

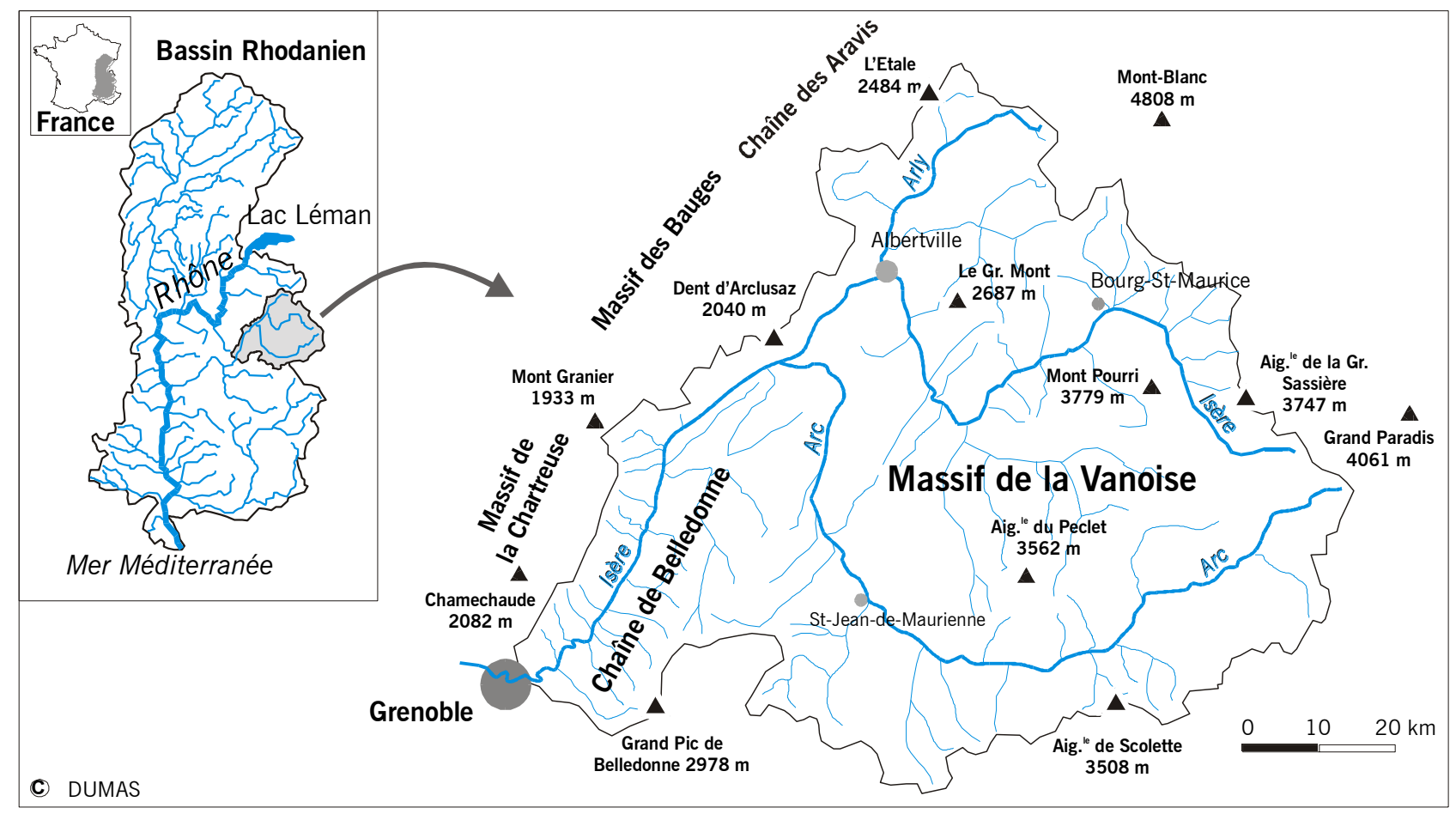

Fig. 1. Localisation et carte du bassin versant de l'Isère à Grenoble. 
Fig. 1. Map of the Isère watershed basin at Grenoble.

\section{Méthodologie}

\subsection{Connaissance systématique des concentrations et des hauteurs d'eau}

Depuis 1993, sur le Campus universitaire de Grenoble, une station hydrométrique fournit, toutes les demiheures, la hauteur et la température de l'eau de l'Isère. Cette station est équipée d'une traille téléphérique permettant d'effectuer régulièrement des jaugeages et d'établir, avec une grande précision, la relation statistique hauteur-débit. Associé à ces mesures, un équipement pour l'étude des flux de matière dissoute (MD) et de matière en suspension (MES) a été installé progressivement depuis 1994 [23]. Actuellement, un préleveur automatique permet d'analyser quotidiennement deux échantillons d'eau sur lesquels sont mesurées les concentrations en MES par filtration-pesée (membranes de 0,45 $\mu \mathrm{m}$ ). Parallèlement à ces prélèvements, des mesures directes en rive gauche, au pas de temps de la demi-heure, sont effectuées par l'intermédiaire de deux sondes turbiditimètriques (Partech IR40C et IR15C).

\subsection{Mesures des concentrations sur la section de l'Isère}

Onze jaugeages complets du débit solide ont été effectués. À ces données ont été ajoutées, après correction, les données issues du jaugeage complet effectué le 04/06/1998 [30]. Tous ces jaugeages ont été réalisés simultanément à un jaugeage du débit liquide par mesure de la vitesse en différents points de la section $[5,7,11,15,18,28]$. En chacun de ces points, un échantillon d'eau (500 ml) est prélevé par aspiration à l'aide d'un préleveur automatique autonome, d'une capacité de 24 flacons, fixé directement sur le câble porteur du téléphérique.

Chaque jaugeage du débit solide se déroule ainsi sur $2 \mathrm{~h}$, durant lesquelles le niveau de l'Isère évolue, et parfois dans des proportions importantes : le 24 avril 2002, l'Isère est montée de près de $50 \mathrm{~cm}$ : le débit est ainsi passé $84 \mathrm{~m}^{3} \mathrm{~s}^{-1}$ au début des mesures à près de $165 \mathrm{~m}^{3} \mathrm{~s}^{-1}$ à la fin du jaugeage (Tableau 1). Ces variations quotidiennes sont bien connues dans les régions alpines et sont liées à la gestion des barrages hydroélectriques. De surcroît, pour l'Isère, ces fluctuations sont amplifiées par la présence de la centrale hydroélectrique du Cheylas, dont les lâchers quotidiens peuvent atteindre $200 \mathrm{~m}^{3} \mathrm{~s}^{-1}$ en l'espace de quelques heures [29]

Tableau 1.

Débits et évolution de l'écoulement pendant les mesures (les valeurs en italique ont été fournies par P. Bois)

Table 1.

Discharges and evolution of the flow during measurements (values in italic were provided by P. Bois)

\begin{tabular}{|c|c|c|c|c|c|c|c|c|c|}
\hline \multirow[t]{2}{*}{ Date } & \multirow{2}{*}{$\begin{array}{l}\mathbf{H}_{\text {réf }} \\
(\mathrm{m})\end{array}$} & \multirow{2}{*}{$\begin{array}{l}\text { Débit } \\
\text { calculé } \\
\left(\mathrm{m}^{3} \mathrm{~s}^{-1}\right)\end{array}$} & \multirow{2}{*}{$\begin{array}{c}\text { Débit } \\
\text { mesuré } \\
\left(\mathrm{m}^{3} \mathrm{~s}^{-1}\right)\end{array}$} & \multirow{2}{*}{$\begin{array}{c}\text { Section } \\
\text { mouillée } \\
\left(\mathrm{m}^{2}\right)\end{array}$} & \multirow{2}{*}{$\begin{array}{c}\text { Vitesse } \\
\text { moyenne } \\
\left(\mathrm{m} \mathrm{s}^{-1}\right)\end{array}$} & \multicolumn{4}{|c|}{ Évolution de l'écoulement } \\
\hline & & & & & & $\begin{array}{c}\text { début du } \\
\text { jaugeage } \\
\left(\mathrm{m}^{3} \mathrm{~s}^{-1}\right)\end{array}$ & $\begin{array}{c}\text { fin du } \\
\text { jaugeage } \\
\left(\mathrm{m}^{3} \mathrm{~s}^{-1}\right)\end{array}$ & $\begin{array}{l}\text { fluctuation du } \\
\text { débit } \\
\text { (écart-type) }\end{array}$ & $\begin{array}{c}\text { tendance } \\
\text { horaire } \\
\left(\mathrm{m}^{3} \mathrm{~s}^{-1} \mathrm{~h}^{-1}\right)\end{array}$ \\
\hline 04/06/1998 & 2,9 & 307,4 & 298,3 & 208,1 & 1,48 & 308,9 & 305,9 & 1,2 & $-1,5$ \\
\hline $17 / 05 / 2001$ & 3,1 & 335,2 & 347,7 & 221,1 & 1,52 & 324,1 & 350,5 & 8,7 & 13,2 \\
\hline 07/02/2002 & 1,6 & 130,0 & 125,5 & 117,9 & 1,10 & 121,8 & 134,5 & 4,5 & 6,4 \\
\hline 01/03/2002 & 1,5 & 116,2 & 114,8 & 109,5 & 1,06 & 120,7 & 107,3 & 4,6 & $-6,7$ \\
\hline 08/03/2002 & 1,6 & 123,0 & 125,0 & 113,9 & 1,08 & 117,3 & 125,2 & 2,5 & 4,0 \\
\hline $14 / 03 / 2002$ & 1,8 & 147,6 & 145,9 & 128,1 & 1,15 & 125,2 & 166,1 & 12,3 & 20,4 \\
\hline $21 / 03 / 2002$ & 2,0 & 178,7 & 176,4 & 144,9 & 1,23 & 178,7 & 202,2 & 8,8 & 11,7 \\
\hline $24 / 04 / 2002$ & 1,6 & 126,4 & 135,8 & 115,9 & 1,09 & 84,1 & 164,8 & 27,1 & 40,4 \\
\hline 02/05/2002 & 1,7 & 136,9 & 138,4 & 122,0 & 1,12 & 138,1 & 135,7 & 0,8 & $-1,2$ \\
\hline $13 / 05 / 2002$ & 2,5 & 240,4 & 239,3 & 176,0 & 1,37 & 221,0 & 246,0 & 7,8 & 12,5 \\
\hline $28 / 06 / 2002$ & 1,9 & 167,3 & 161,9 & 138,5 & 1,21 & 171,1 & 163,6 & 2,3 & $-3,8$ \\
\hline $10 / 10 / 2002$ & 1,5 & 113,9 & 105,1 & 108,3 & 1,05 & 119,5 & 114,0 & 2,5 & $-2,8$ \\
\hline 09/06/1998 & 2,7 & 265,0 & - & 186,9 & 1,42 & 259,0 & 271,0 & 4,8 & 6,0 \\
\hline $14 / 06 / 1998$ & 1,8 & 139,0 & - & 122,7 & 1,13 & 141,0 & 137,0 & 1,4 & $-2,0$ \\
\hline $17 / 06 / 1998$ & 2,2 & 198,0 & - & 152,8 & 1,30 & 183,0 & 214,0 & 12,1 & 15,5 \\
\hline 22/06/1998 & 2,5 & 228,0 & - & 168,1 & 1,36 & 211,0 & 240,0 & 11,1 & 14,5 \\
\hline 04/07/1998 & 2,3 & 201,0 & - & 154,3 & 1,30 & 198,0 & 205,0 & 2,9 & 3,5 \\
\hline
\end{tabular}


En chacun des points de prélèvement, la hauteur instantanée de l'Isère est notée, afin de pouvoir caler précisément sur le profil transversal, les points de mesure en fonction de la cote retenue. Par ailleurs, afin de déterminer une hauteur de l'Isère représentative au moment du jaugeage, une cote de référence $\left(H_{\text {réf }}\right)$ est calculée en fonction des hauteurs moyennes mesurées à chaque verticale pondérées par la surface des paraboles des vitesses observées (profils unitaires des vitesses, $\mathrm{PU}$, en $\mathrm{m}^{2} \mathrm{~s}^{-1}$ ).

\subsection{Le profil du fond du lit de l'Isère}

La détermination des concentrations nous a, dans un premier temps, contraints à préciser le profil transversal de l'Isère à la hauteur de la station hydrométrique. Les mesures des profondeurs, collectées directement lors des jaugeages, montrent des dissemblances suffisamment fortes pouvant biaiser significativement l'estimation des concentrations moyennes. Les profils bathymétriques déterminés à partir des jaugeages montrent ainsi à chaque fois des superficies légèrement différentes liées non pas à une mobilité importante du fond (la relation hauteur-débit n'a d'ailleurs pas varié depuis 1996), mais directement à l'incertitude de la détection du fond du lit. Si cette imprécision n'a que très peu d'incidence sur la mesure du débit puisque les vitesses diminuent très fortement au niveau du fond; ce n'est pas le cas pour les concentrations qui peuvent présenter des valeurs importantes sur les bordures. Aussi, afin de déterminer un profil moyen de référence, nous avons utilisé les 46 jaugeages effectués depuis 1996. Ils totalisent plus de 430 mesures du fond.

\subsection{Spatialisation des mesures ponctuelles}

Les données issues de six jaugeages complets effectués en 1998 ont été en partie intégrées dans cette étude [30]. Mais, excepté pour le jaugeage du 4 juin 1998, les données brutes n'ont pas été conservées. Au total, douze jaugeages complets ont permis de reconnaître la répartition de la charge en suspension totale dans la section transversale de l'Isère. Les vitesses et les concentrations présentent dans l'ensemble une forte dépendance spatiale. Une analyse des variogrammes calculés pour deux directions perpendiculaires l'une à l'autre montre qu'un krigeage linéaire, avec un coefficient d'anisotropie de 13, s'ajuste bien à l'ensemble des données et permet de compenser la forte asymétrie de la section mouillée, dont la largeur est de 15 à 30 fois plus importante que la profondeur moyenne. En outre, cette spatialisation a été vérifiée et contrôlée avec une comparaison des cartes des vitesses, dont le calcul du volume représente le débit instantané (Tableau 1 : débit mesuré), et la valeur du débit calculée directement avec la relation hauteurdébit (relation fournie par $P$. Bois : $Q=65,441 \mathrm{H}^{1,439}$ ). L'erreur reste toujours inférieure à $6 \%$ et le plus souvent cantonnée à $3 \%$. Pour cette étude, afin de conserver une homogénéité des résultats, les flux de MES sont déterminés à partir des débits calculés avec la courbe de tarage de la station.

\section{Présentation des résultats}

\subsection{Répartition des matières en suspension au sein de la section}

Les profils de MES obtenus sont extrêmement variables et ne montrent pas toujours des concentrations de matières en suspension plus importantes au voisinage du fond. On retrouve, d'une manière plus ou moins nette, quatre familles différentes de répartition des concentrations (Fig. 2).

Ces répartitions verticales ou latérales sont souvent décrites et s'expliquent notamment par la texture des sédiments en suspension $[3,8,9]$. On oppose généralement deux modes de répartition : une suspension uniforme lorsque les sédiments ont une texture argileuse et, à l'inverse, une suspension graduée lorsque les sédiments deviennent plus limoneux. La vitesse du courant pourrait donc en partie contrôler ces gradients de turbidité, puisque la plupart des modèles de transport de sédiments montrent l'importance de seuils de cisaillement et des vitesses pour que soit mis en mouvement un élément d'un diamètre donné $[4,12,13,20$, 24].

À ce jour, la connaissance de ces répartitions et de leur variabilité temporelle reste souvent très sommaire. Aussi, sur certains cours d'eau, on considère que les gradients de turbidité sont faibles, voire homogènes. On utilise alors directement la valeur issue d'un prélèvement unique pour déterminer les flux globaux de MES $[14,26]$. 

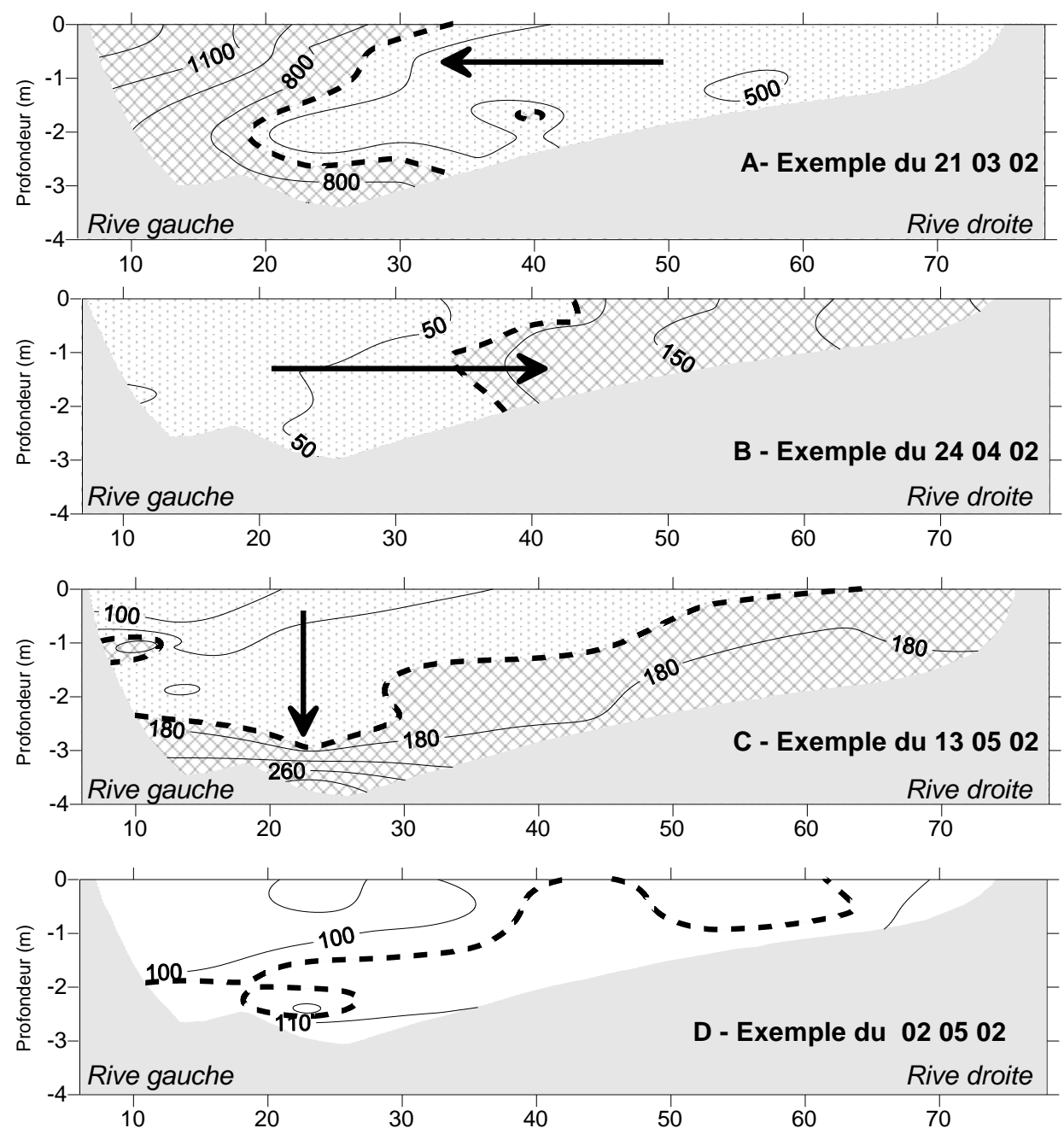

Fig. 2. Répartition de la charge en suspension totale, dans la section transversale de l'Isère : (A) gradient latéral négatif, (B) gradient latéral positif, (C) suspension graduée verticale, (D) suspension uniforme. Le trait discontinu représente la courbe égale à la concentration moyenne de la section, la trame ponctuelle la zone de sous-estimation, et la trame quadrillée la zone de surestimation.

Fig. 2. Total suspended load distribution in the transverse section of Isère : (A) negative lateral gradient; (B) positive lateral gradient; (C) vertical graduated suspension, (D) uniform suspension. The discontinuous line represents the curve equal to the section average concentration, the dotted mesh the zone of underestimation, and the squared mesh the zone of overestimation.

Pour quantifier le plus précisément possible ces flux de matière, il est en revanche utile de chercher des paramètres pouvant intervenir dans le passage d'un mode de répartition des concentrations à un autre. Sur I'Isère, cette connaissance contribuera, entre autres, à une meilleure estimation de la concentration moyenne $\left(C_{\mathrm{m}}\right)$ et du flux de MES associé $\left(Q_{\mathrm{s}}\right)$. Lorsque le débit solide est approché à partir d'un seul et unique prélèvement, situé par exemple sur la rive gauche du cours d'eau $\left(M E S_{\text {fixe }}\right)$, la valeur enregistrée peut être parfois assez proche de la concentration moyenne, mais parfois nettement sous-estimée ou au contraire surestimée (Fig. 2). Il est donc peu sûr de corriger les valeurs mesurées ponctuellement directement à l'aide d'un modèle régressif unique, même si ce dernier présente, dans le cas de l'Isère, un bon coefficient de corrélation. Cette méthode de correction est parfois utilisée et permet sans aucun doute d'améliorer l'extrapolation des valeurs ponctuelles [2, 19]. Sur l'Isère, l'ajustement statistique correspond alors à une droite (équation (1)) :

$\mathrm{C}_{\mathrm{m}}=0,832 \mathrm{MES}_{\mathrm{fixe}}+18,46 \quad \mathrm{R}^{2}=0,94$

La qualité statistique de cette relation démontre qu'il reste possible d'appréhender le flux réel de MES à partir d'une mesure unique, sous réserve d'appliquer une correction limitant la surestimation, ou la sousestimation, liée aux particularités du site de mesure.

Toutefois, le bon coefficient de corrélation de cette relation est lié à la grande variabilité naturelle des concentrations, inscrites dans une large gamme de valeurs allant de 20 à plus de $1000 \mathrm{mg} \mathrm{I}^{-1}$. Le coefficient 
de Bravais-Pearson devient plus faible en prenant en compte les valeurs inférieures à $600 \mathrm{mg} \mathrm{l}^{-1}\left(\mathrm{R}^{2}=0,75\right)$ qui représentent plus de $70 \%$, voire plus de $90 \%$ suivant les années, des mesures annuelles.

Par ailleurs, on peut, à la lecture de ces premiers résultats et de l'expérience acquise, pressentir qu'une suspension graduée latérale droite ou gauche puisse apparaître dans toutes les gammes de concentration. Aussi il n'est pas exclu de retrouver, même pour des concentrations élevées, les rapports $C_{\mathrm{m}} / \mathrm{MES}_{\mathrm{fixe}}$ observés. Le ratio le plus faible est égal à 0,56 et le plus élevé à 1,75 . La concentration moyenne de la section $\left(C_{\mathrm{m}}\right)$ pourrait donc osciller entre $-44 \%$ et $+75 \%$ de la valeur observée sur la rive gauche et donc varier dans un rapport de 1 à 3 . II est important dès lors de chercher à minimiser le plus possible cette incertitude.

\subsection{Détermination des gradients et extrapolation des mesures ponctuelles}

Pour chaque jaugeage complet, nous avons décrit la répartition des concentrations à partir de différents paramètres statistiques issus des caractéristiques hydrodynamiques et hydrosédimentaires de l'Isère (Tableau 2).

Tableau 2

Mesures complètes de la MES sur la section de l'Isère-Campus

Table 2.

SPM complete measurements on the Isère-Campus section

\begin{tabular}{|c|c|c|c|c|c|c|c|c|c|c|c|}
\hline \multirow[t]{3}{*}{ Date } & \multicolumn{2}{|c|}{ Mesure ponctuelle } & \multicolumn{8}{|c|}{ Matière en suspension sur l'ensemble de la section } & \multirow{3}{*}{$\begin{array}{l}\text { Débit } \\
\text { solide } \\
\left(\mathrm{kg} \mathrm{s}^{-1}\right)\end{array}$} \\
\hline & \multirow{2}{*}{$\begin{array}{l}\text { moyenne } \\
\left(\mathrm{mg} \mathrm{l}^{-1}\right)\end{array}$} & \multirow{2}{*}{$\begin{array}{l}\text { tendance } \\
\left(\mathrm{mg} \mathrm{l}^{-1} \mathrm{~h}^{-1}\right)\end{array}$} & \multirow{2}{*}{$\begin{array}{l}\text { Concentration } \\
\text { moyenne } \\
\left(\mathrm{mg}^{-1}\right)\end{array}$} & \multirow{2}{*}{$\begin{array}{l}\text { Valeur } \\
\text { mini } \\
\left(\mathrm{mg} \mathrm{I}^{-1}\right)\end{array}$} & \multirow{2}{*}{$\begin{array}{l}\text { Valeur } \\
\text { maxi } \\
\left(\mathrm{mg} \mathrm{l}^{-1}\right)\end{array}$} & \multirow{2}{*}{$\begin{array}{l}\text { Écart } \\
\text { absolu } \\
\text { max- } \\
\text { mini }\end{array}$} & \multirow{2}{*}{$\begin{array}{l}\text { Écart } \\
\text { type }\end{array}$} & \multicolumn{3}{|c|}{ Gradient ( $\mathrm{mg} \mathrm{l}^{-1} \mathrm{~m}^{-1}$ ) } & \\
\hline & & & & & & & & $\begin{array}{l}\text { sur } \\
\text { l'axe } \\
\text { des } X\end{array}$ & $\begin{array}{l}\text { sur } \\
\text { l'axe } \\
\text { des } Y\end{array}$ & $R^{2}$ & \\
\hline 04/06/1998 & 1481,8 & 15,9 & 1396,2 & 1060 & 1959 & 900 & 262 & 10,3 & $-618,0$ & 0,47 & 429,1 \\
\hline $17 / 05 / 2001$ & 694,2 & 0,8 & 390,3 & 317 & 754 & 436 & 96 & 0,4 & 2,0 & 0,70 & 130,8 \\
\hline 07/02/2002 & 30,5 & 0,3 & 53,3 & 28 & 161 & 133 & 29 & 0,4 & $-20,2$ & 0,22 & 6,9 \\
\hline 01/03/2002 & 113,6 & $-1,8$ & 109,5 & 60 & 137 & 76 & 25 & $-1,0$ & $-1,6$ & 0,82 & 12,7 \\
\hline 08/03/2002 & 26,2 & $-0,1$ & 23,6 & 14 & 90 & 76 & 17 & 0,2 & $-17,4$ & 0,47 & 2,9 \\
\hline $14 / 03 / 2002$ & 36,4 & 1,5 & 48,1 & 16 & 93 & 77 & 18 & 1,2 & $-8,4$ & 0,89 & 7,1 \\
\hline $21 / 03 / 2002$ & 1008,3 & $-18,6$ & 706,2 & 498 & 1111 & 613 & 151 & $-7,7$ & 6,3 & 0,51 & 126,2 \\
\hline $24 / 04 / 2002$ & 62,7 & 4,1 & 86,4 & 24 & 224 & 200 & 60 & 3,7 & $-2,2$ & 0,92 & 10,9 \\
\hline $02 / 05 / 2002$ & 99,9 & 0,1 & 104,1 & 94 & 129 & 35 & 7 & 0,3 & $-6,5$ & 0,47 & 14,2 \\
\hline $13 / 05 / 2002$ & 197,8 & 3,2 & 168,5 & 88 & 328 & 240 & 43 & 1,0 & $-34,1$ & 0,59 & 40,5 \\
\hline 28/06/2002 & 117,7 & $-0,7$ & 89,6 & 70 & 133 & 63 & 20 & $-0,8$ & $-5,1$ & 0,62 & 15,0 \\
\hline $10 / 10 / 2002$ & 59,8 & $-0,5$ & 36,6 & 17 & 76 & 58 & 18 & $-0,8$ & $-1,8$ & 0,63 & 4,2 \\
\hline 09/06/1998 & 306 & & 532,0 & & & & & & & & 141,0 \\
\hline $14 / 06 / 1998$ & 134 & & 192,0 & & & & & & & & 26,7 \\
\hline $17 / 06 / 1998$ & 140 & & 189,0 & & & & & & & & 37,4 \\
\hline $22 / 06 / 1998$ & 160 & & 212,0 & & & & & & & & 48,3 \\
\hline $04 / 07 / 1998$ & 614 & & 619,0 & & & & & & & & 124,4 \\
\hline
\end{tabular}

La valeur des gradients horizontaux et verticaux est calculée à partir des coefficients d'une surface de tendance plane (spleen du $1^{\text {er }}$ degré) s'ajustant aux valeurs ponctuelles. A partir des deux gradients ainsi déterminés, le type de répartition des concentrations au sein de la section peut être correctement approché d'une manière synthétique avec le rapport suivant (2) :

Ratiogradients $=\frac{\text { Gradient } X}{\mid \text { Gradient } Y \mid}$

Le signe de ce ratio est donné par l'orientation du gradient latéral, puisque c'est ce dernier qui favorise ou non une surestimation de la concentration à partir d'un échantillon prélevé depuis une berge. Le signe du gradient vertical est, en effet, peu significatif. Sur cet axe, les gradients positifs faibles indiquent, avant tout, une suspension largement dominée par une suspension latérale.

La centrale hydroélectrique du Cheylas, située à environ $30 \mathrm{~km}$ en amont de la station, avec des restitutions hydrologiques biquotidiennes en rive gauche, pourrait peut-être expliquer les modifications des gradients de turbidité. II possible également que les méandres successifs précédant la station hydrométrique favorisent, en fonction de certaines situations hydrologiques et hydrodynamiques, des répartitions particulières de la concentration. En revanche, le passage d'un mode de suspension à un autre n'est pas expliqué par des variations nettes de la répartition des champs de vitesse au sein de la section. Le tracé des courbes 
isotaches reste très stable et montre des vitesses maximales invariablement placées, non loin de la surface, entre 40 et $50 \mathrm{~m}$ de la rive gauche.

L'origine des eaux écoulées (pluviale, nivale, pluvio-nivale...) est, à ce stade, encore difficile à déterminer avec précision. Mais une séparation des eaux au sein de la section mouillée n'est guère envisageable, car la minéralisation des eaux présente toujours une très grande homogénéité spatiale.

Différents modèles régressifs multivariés ont été testés afin de cerner les variables susceptibles d'expliquer au mieux ce paramètre. Trois variables jouent, d'une manière hautement significative $\left(R^{2}=0,88\right)$, sur la nature de la répartition des concentrations au sein de la section (équation (3)).

Ratio $_{\text {gradients }}=0,034 \mathrm{~T}_{\mathrm{Q}}+0,061 \mathrm{~T}_{\mathrm{MES}}-0,869 \mathrm{~V}_{\mathrm{m}}+0,739$

$T_{Q}$ étant la variation du débit, $T_{M E S}$ la variation horaire moyenne des concentrations et $V_{m}$ la vitesse moyenne instantanée de l'eau.

L'équation (3) indique qu'une hausse du débit, et/ou une augmentation de la concentration, facilitent l'apparition d'un gradient latéral positif (les concentrations augmentent en allant vers la rive droite). Ce gradient sera d'autant plus accentué que ces variations coïncident avec un débit réduit, puisque la vitesse moyenne de l'écoulement est alors plus faible. C'est sans doute la conséquence d'une influence plus marquée des restitutions du Cheylas sur l'hydrodynamique de l'écoulement lorsque le niveau de l'Isère est bas. A l'inverse, les effets des lâchers sont atténués lorsque le débit initial est plus important.

Dans la mesure où ces trois variables interviennent dans l'explication des variations spatiales des concentrations, nous avons choisi de les intégrer dans un modèle linéaire. La concentration moyenne réelle au sein de la section est alors approchée à partir de l'équation (4) :

$\mathrm{C}_{\mathrm{m}}=0,926 \mathrm{MES}_{\mathrm{fixe}}-0,445 \mathrm{~T}_{\mathrm{Q}}+10,539 \mathrm{~T}_{\mathrm{MES}}-446,41 \mathrm{~V}_{\mathrm{m}}+502,2$

Au-delà d'un coefficient de corrélation élevé $\left(R^{2}=0,987\right)$, la prise en compte de la dynamique hydrologique et des concentrations, aisée à appréher avec des mesures en continu, permet d'envisager une quantification

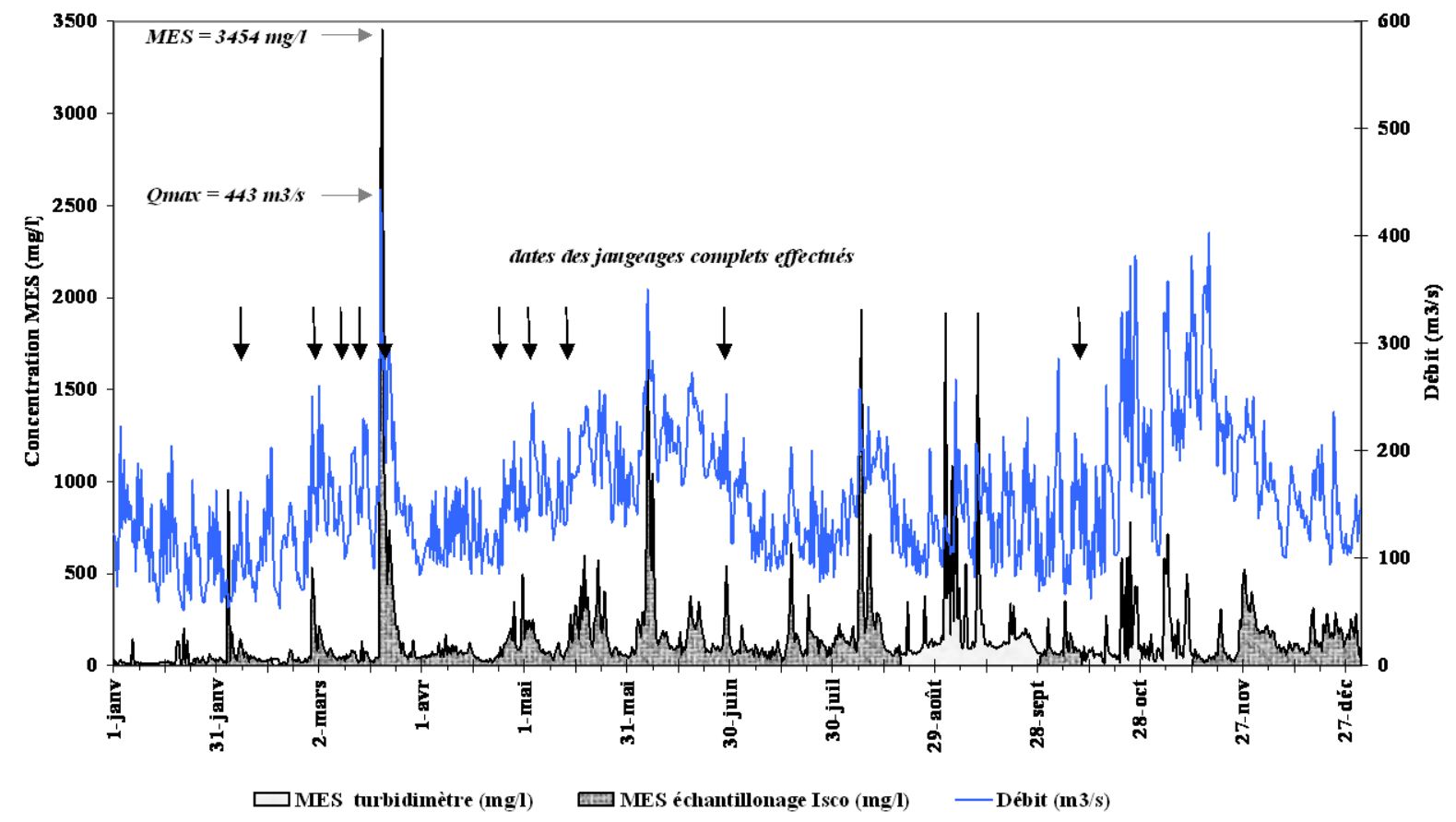

plus robuste des flux de MES à partir d'un prélèvement unique.

Fig. 3. Evolution des concentrations mesurées en rive gauche par échantillonnage d'eau et à partir des turbidimètres (exemple de l'année 2002).

Figure 3. Evolution of the concentrations measured on left bank by water sampling and from the turbidimeters (example of the year 2002). 


\section{Estimation des flux MES corrigés par différentes méthodes}

Nous avons calculé la quantité annuelle de sédiments véhiculée par l'Isère au niveau de Grenoble (Tableau 3). Les quantités sont estimées à partir de données biquotidiennes de l'écoulement et des concentrations ponctuelles mesurées en rive gauche (exemple de solidigramme pour l'année 2002 sur la Fig. 3).

Le flux de MES est déterminé, d'une part, directement à partir des mesures ponctuelles (prélèvements en rive gauche) et, d'autre part, à partir des relations déterminées précédemment (équations (1) et (4)). Dans certains cas de figure - concentrations ponctuelles très faibles (inférieures à $20 \mathrm{mg} \mathrm{l}^{-1}$ ) couplées à des conditions hydrodynamiques particulières-, l'équation (4) appliquée directement aux données fournit des valeurs parfois légèrement négatives. Ces cas représentent moins de $5 \%$ des valeurs annuelles. Nous avons alors remplacé ces valeurs négatives par des valeurs nulles, puis égales à $30 \mathrm{mg} \mathrm{I}^{-1}$, ce qui représente la moyenne des valeurs les plus faibles. Cette valeur semble d'ailleurs plus réaliste qu'une valeur nulle, jamais observée en rive gauche. De surcroît, le résultat final à l'échelle annuelle n'est que très faiblement affecté par le choix de cette valeur seuil (Tableau 3).

L'analyse des résultats montre clairement que l'évaluation des flux annuels est fortement tributaire de la méthode d'extrapolation retenue. Les valeurs extrêmes envisageables (mini et maxi) ne sont qu'indicatives et s'éloignent probablement de la réalité. On reste néanmoins très loin du chiffre de $19,9 \mathrm{Mt} \mathrm{an}^{-1}$, estimé pour I'Isère à Grenoble par Müntz et Lainé [16, 17], puis repris par Pardé [21] dans sa thèse. Cette valeur est cependant jugée, quelques années après, comme invraisemblable par Pardé [22].

L'utilisation directe de la mesure ponctuelle pour estimer les flux de MES engendre très probablement une surestimation globale des quantités annuelles. Cette surestimation des mesures ponctuelles avait déjà été perçue et expliquée à la fois par la position du point de prélèvement, placée dans une concavité du chenal, et une altitude indépendante de la ligne d'eau [23]. Lors des hautes eaux l'échantillonnage s'effectue davantage au fond où les concentrations sont généralement plus fortes. La plus forte surestimation des quantités transportées évaluées directement avec la mesure ponctuelle s'observe en 1999, avec $470000 \mathrm{t}$ par rapport à la valeur issue de l'équation (1) et près de $950000 \mathrm{t}$ avec l'équation (4), soit une différence qui correspond respectivement à un flux spécifique de $84 \mathrm{t} \mathrm{km}^{-2} \mathrm{an}^{-1}$ et de $170 \mathrm{t} \mathrm{km}^{-2} \mathrm{an}^{-1}$. Ces valeurs sortent de la fourchette des dégradations spécifiques moyennes européennes comprises entre 30 et $90 \mathrm{t} \mathrm{km}^{-2} \mathrm{an}^{-1}$ [27]. Elles montrent bien la grande difficulté d'apprécier ces flux sur un cours d'eau important comme l'Isère, fortement chargé en sédiments. Mais ce constat est sans doute applicable à bien des cours d'eau alpins.

Tableau 3

Détermination annuelle des flux de MES de l'Isère à Grenoble

Table 3.

Annual determination of SPM flows of Isère in Grenoble

Écoulement MES (tonnes) : évaluation

\begin{tabular}{ccccccc}
\hline $\begin{array}{c}\text { Modules } \\
\left(\mathrm{m}^{3} \mathrm{~s}^{-1}\right)\end{array}$ & $\begin{array}{c}\text { Volume d'eau } \\
\text { écoulée } \\
\left(\mathrm{Mm}^{3}\right)\end{array}$ & $\begin{array}{c}\text { À partir du } \\
\text { prélèvement } \\
\text { en rive } \\
\text { gauche }\end{array}$ & $\begin{array}{c}\text { Valeurs } \\
\text { minimales }\end{array}$ & $\begin{array}{c}\text { Valeurs } \\
\text { maximales }\end{array}$ & $\begin{array}{c}\text { Équation (1) } \\
\text { envageables envisageables }\end{array}$ & $\begin{array}{c}\text { Équation (4), avec les } \\
\text { valeurs négatives égales } \\
\text { à : }\end{array}$ \\
& & & $0 \mathrm{mg} \mathrm{l}^{-1}$ & $30 \mathrm{mg} \mathrm{l}^{-1}$
\end{tabular}

\begin{tabular}{llrrrrrrr}
\hline $\mathbf{1 9 9 6}$ & 140.0 & 4429 & $\mathbf{1 2 8 1 3 1 4}$ & 717536 & 2242300 & 1147811 & 1017478 & $\mathbf{1 0 2 4} \mathbf{2 0 9}$ \\
$\mathbf{1 9 9 7}$ & 155.6 & 4906 & $\mathbf{1 0 7 4 0 9 4}$ & 601493 & 1879665 & 984221 & 738193 & $\mathbf{7 5 4} \mathbf{3 0 9}$ \\
$\mathbf{1 9 9 8}$ & 143.7 & 3016 & $\mathbf{6 7 7 2 8 0}$ & 379277 & 1185240 & 619183 & 540344 & $\mathbf{5 4 5 0 0 8}$ \\
$\mathbf{1 9 9 9}$ & 206.2 & 65021 & $\mathbf{3 5 3 3 \mathbf { 3 7 8 }}$ & 1978692 & 6183412 & 3059797 & 2558503 & $\mathbf{2 5 8 4} \mathbf{1 6 3}$ \\
$\mathbf{2 0 0 0}$ & 166.8 & 5276 & $\mathbf{1 2 6 3 9 7 7}$ & 707827 & 2211960 & 1149025 & 903409 & $\mathbf{9 2 3} \mathbf{1 5 1}$ \\
$\mathbf{2 0 0 1}$ & 209.6 & 6609 & $\mathbf{3 3 0 7 8 5 5}$ & 1852399 & 5788746 & 2874142 & 2437559 & $\mathbf{2 4 8 9 0 2 8}$ \\
$\mathbf{2 0 0 2}$ & 155.9 & 4917 & $\mathbf{9 4 5} \mathbf{4 4 3}$ & 529448 & 1654525 & 877388 & 671715 & $\mathbf{6 9 2} \mathbf{9 1 4}$
\end{tabular}

* L'année 1998 est incomplète : les valeurs sont issues d'observations couvrant la période du $1^{\text {er }}$ janvier au 31 août.

*The year 1998 is incomplete: the values result from observations covering the period from 1 January to 31 August.

Les flux de MES montrent une grande variabilité interannuelle, selon l'hydraulicité de l'Isère. Si l'on admet que l'équation (4) limite l'imprécision, les valeurs s'échelonnent de $690000 \mathrm{t}$ en 2002, à près de 2,6 Mt en 1999. Le flux spécifique de l'Isère à Grenoble varie ainsi entre 124 et plus de $460 \mathrm{t} \mathrm{km}^{-2} \mathrm{an}^{-1}$

Sur les six années complètes étudiées, le flux moyen est d'environ 1,4 Mt $\mathrm{an}^{-1}$. Cependant, la grande variabilité interannuelle de ce flux oblige à conserver une certaine prudence dans l'estimation d'une valeur annuelle représentative. De plus, la période étudiée présente à plusieurs reprises des modules plus faibles 
que le module moyen de l'Isère $\left(200 \mathrm{~m}^{3} \mathrm{~s}^{-1}\right)$. Dans la mesure où le flux de MES est fortement corrélé au module annuel $\left(R^{2}=0,88\right)$, il est possible de l'évaluer pour une année hydrologique moyenne. Cette relation s'explique néanmoins par le fait que le débit solide annuel est aussi, dès l'origine, calculé en intégrant la valeur du débit liquide.

En année moyenne, l'Isère exporterait $2,2 \mathrm{Mt} \mathrm{an}^{-1}$ de sédiments en suspension, ce qui représente presque la moitié, voire le tiers, du débit solide annuel moyen du Rhône à son embouchure $\left(5\right.$ à $\left.6 \mathrm{Mt} \mathrm{an}^{-1}\right)$ [26]. En moyenne, le flux spécifique de l'Isère serait donc d'un peu moins de $400 \mathrm{t} \mathrm{km}^{-2} \mathrm{an}^{-1}$. Ce taux d'érosion spécifique est considérable et dépasse même celui de la Durance évalué à $360 \mathrm{t} \mathrm{km}^{-2} \mathrm{an}^{-1}$ [1]. Ces valeurs restent, pour ces latitudes, très élevées. Elles dépassent également légèrement la zonation de 250 à $350 \mathrm{t}$ $\mathrm{km}^{-2} \mathrm{an}^{-1}$ proposée pour la bordure est-pyrénéenne $[28,29]$.

\section{Conclusion}

Avec des taux d'érosion spécifiques très élevés et une forte variabilité temporelle des concentrations, l'Isère demeure un témoin particulièrement révélateur du fonctionnement hydrosédimentaire des grands cours d'eau alpins. Le contexte montagneux de son bassin, son module important et son régime perturbé par de nombreux aménagements hydroélectriques engendrent, au cours d'une année, des flux de MES marqués par une succession de pics. Ces fortes fluctuations temporelles s'accompagnent simultanément d'une grande variabilité des gradients de turbidité au sein de la section de cette rivière. Aussi la quantification des flux sur ce type de cours d'eau est extrêmement délicate. II est cependant rarement possible, et plus encore sur des cours d'eau importants, de prélever une dizaine d'échantillons dans la section de manière systématique, au pas de temps de $12 \mathrm{~h}$, voire quotidiennement. La prise en compte de paramètres hydrologiques et de la dynamique des flux de MES améliore sensiblement la connaissance des répartitions et offre ainsi une extrapolation plus robuste d'un unique point de mesure, qui selon les cas, sous-estime ou surestime la concentration moyenne.

\section{Remerciements}

De 1999 à 2001, ce travail a bénéficié d'une aide financière recueillie dans le cadre d'un programme financé par la Communauté Européenne : FLOodplain Biodiversity And Restoration (FLOBAR 2). L'auteur remercie $P$. Bois (professeur à l'université de Grenoble), M. Mietton (professeur à l'université de Lyon) et tout particulièrement J.-L. Peiry (professeur à l'université de Clermont-Ferrand) pour lui avoir transmis des données ainsi qu'une partie de l'équipement de la station Grenoble-Campus.

\section{Références}

[1] P. Alary, Mécanismes et bilans d'érosion dans un bassin versant méditerranéen aménagé : le cas de la Durance (S-E France). Thèse, Univ. d'Aix-Marseille, 1998.

[3] J. Brasington, K. Richards, Turbidity and suspended sediment dynamics in small catchments in the Nepal Middle Hills, Hydrological Processes 14 (2000) 2559-2574.

[4] J.-P. Bravard, F. Petit, Les cours d'eau. Dynamique du système fluvial, Armand Colin, Masson, 1997.

[5] I. Cojan, M. Renard, Sedimentologie, Masson, Paris, 1997.

[6] J. Colombani, Contribution à la méthodologie des mesures systématiques de débits solides en suspension, Cah. Orstom, sér. Hydrol. IV (2) (1967) 27-36.

[7] W.F. Curtis, R.H. Meade, C. Nordin, N.B. Price, Z.R. Sholkovitz, Non-uniform vertical distribution of fine sediment in the Amazone river, Nature 280 (1979) 381-383.

[8] H.D. (don) Duizendstra, Determination of sediment transport in an armoured grave-bed river, Earth Surface Processes Landforms 26 (2001) 1381-1393.

[9] D. Eisma, Suspended matter in the aquatic environment, Springer-Verlag, Berlin, 1993.

[10] M. Felix, Flow structure of turbidity currents, Sedimentology 49 (3) (2002) 397-413.

[11] R. Girard, La matière en suspension de l'Isère à Grenoble, mémoire de maîtrise, Institut de Géographie Alpine, Grenoble, 2002.

[12] J.-L. Guyot, J.-M. Jouanneau, J. Quintanilla, J.G. Wasson, Les flux de matières dissoutes et particulaires exportés des Andes par le Rio Béni (Amozonie bolivienne), en période de crue, Geodin. Acta 6 (4) (1993) 233-241. 
[13] F. Hjulstrom, Studies of the morphological activity of rivers as illustrated by the the River Fyros, Bull. Geol. Inst. Uppsala 25 (1935) 221-527.

[14] J. Larras, Hydraulique et granulats, Eyrolles, Paris, 1972.

[15] M. Meddi, Etude du transport solide dans le bassin versant de l'oued Ebda (Algérie), Z. Geomorphol. 43 (2) (1999) 167-183.

[16] M. Mietton, Les processus élémentaires d'érosion hydrique à l'échelle du versant, in : L'érosion entre nature et société, Éditions Sedes, 1998, pp. 57-68.

[17] A. Müntz, E. Lainé, Les matériaux charriés par les cours d'eau des Alpes et des Pyrénées, C. R. Acad. Sci. Paris 156 (1913) 848-851.

[18] A. Müntz, E. Lainé, Etudes sur la formation des limons et leurs charriages par les cours d'eau des Alpes et des Pyrénées, C. R. Acad. Sci. Paris 160 (1915) 462-467.

[19] J.-F. Nouvelot, Mesure et étude des transports solides en suspension au Cameroun, Cah. Orstom, sér. Hydrol. VI (4) (1969) 43-56.

[20] J.-F. Nouvelot, Méthodologie pour la mesure en réseau des transports solides en suspension dans les cours d'eau intertropicaux peu chargés, Cah. Orstom, sér. Hydrol. IX (1) (1972) 3-18.

[21] C. O'Riordan, M.-A. Maldiney, J.-M. Mouchel, M. Poulin, Un nouveau dispositif d'analyse du transport des matières en suspension dans les rivières, C. R. Acad. Sci. Paris, Sér. Ila 322 (1996) 285-291.

[22] M. Pardé, Le régime du Rhône. Étude hydrologique, in : Etudes et Travaux, vol. 1, université de Lyon, Institut des Etudes rhodaniennes, 1925.

[23] M. Pardé, Quelques nouveautés sur le régime du Rhône, in : Mém. et Doc., vol. 1, université de Lyon, Institut des études rhodaniennes, 1942.

[24] J.-L. Peiry, Recherches en géomorphologie fluviale dans les hydrosystèmes fluviaux des Alpes du Nord, IGA Habilitation, université Joseph-Fourier, Grenoble-1, 1997.

[25] F. Petit, Phénomènes influençant la mise en mouvement et le transport des particules en rivières naturelles, Z. Geomorphol. 32 (3) (1988) 299-310.

[26] A. Pont, Les débits solides du Rhône à proximité de son embouchure : données récentes (1994-1995), Rev. Géogr. de Lyon vol. 72 (1) (1997) 23-33.

[28] P. Serrat, Dynamique sédimentaire actuelle d'un système fluvial méditerranéen : I'Agly (France), C. R. Acad. Sci. Paris, Ser. Ila 329 (3) (1999) 189-196.

[29] P. Serrat, W. Ludwig, B. Navarro, J.-L. Blazi, Variabilité spatio-temporelle des flux de matière en suspension d'un fleuve côtier méditerranéen : la Têt (France), C. R. Acad. Sci. Paris, série lla 333 (2001) 389-397.

[30] P. Touchebeuf de Lussigny, Mesures de transports solides en suspension effectuées par l'Orstom en Afrique Noire, Orstom, 1970.

[31] F. Vautier, Dynamique géomorphologique et végétalisation des cours d'eau endigués : l'exemple de I'Isère dans le Grésivaudan, thèse, Institut de Géographie alpine, Grenoble, 2000.

[32] $S$. Veyrat, Etude de la répartition des concentrations de MES sur une section de I'Isère (station de Grenoble-Campus), mémoire de DEA, Institut de Géographie alpine, Grenoble, 1998.

[33] D.E. Walling, P. Kane, Temporal variation of suspended sediment properties, IAHS-AISH / Int. Assoc. Hydrol. Sci. 137 (1982) 409-417. 